\title{
Association of single-nucleotide polymorphisms in antioxidant genes and their gene-gene interactions with risk of male infertility in a Chinese population
}

\author{
YULING YIN $^{1 *}$, PEIRAN ZHU ${ }^{2 *}$, TAO LUO ${ }^{2}$ and XINYI XIA ${ }^{1,2}$ \\ ${ }^{1}$ Research Institute of Life Sciences, Jiangsu University, Zhenjiang, Jiangsu 212013; \\ ${ }^{2}$ Department of Reproduction and Genetics, Institute of Laboratory Medicine, Jinling Hospital, \\ Nanjing University School of Medicine, Nanjing, Jiangsu 210002, P.R. China
}

Received September 30, 2019; Accepted April 7, 2020

DOI: $10.3892 /$ br.2020.1306

\begin{abstract}
The antioxidant defense system protects DNA from the damaging effects of oxidative stress and is hypothesized to be associated with an increased risk of male infertility. Polymorphisms in antioxidant genes and the gene-gene interactions associated with the antioxidant system may increase the potential risk of male infertility. In the present case-controlled study, the individual link between seven gene polymorphisms (NQO1 rs1800566, SOD2 rs4880, GSTM3 rs1571858, rs3814309, rs7483, GSTM5 rs11807 and GSTP1 rs1695) and the risk of male infertility was investigated. A total of 248 idiopathic infertility patients and 310 fertile controls were selected, and genotyping was performed using the Mass ARRAY platform. There were no significant associations between the seven polymorphisms and risk of male infertility. However, the analysis of gene-gene interactions showed a decreased risk of male infertility in GSTM3 rs3814309/NQO1 rs1800566 [CC x CT/TT; odds ratio $(\mathrm{OR})=0.56,95 \%$ confidence interval $(\mathrm{CI})=0.34-0.92 ; \mathrm{P}=0.022)$, and a significant association between a gene-gene interaction in GSTM3 rs1571858/NQO1 rs1800566 and azoospermia (AG/GG x CC; $\mathrm{OR}=3.84$, $95 \% \mathrm{CI}=1.25-11.81 ; \mathrm{P}=0.019)$.
\end{abstract}

\section{Introduction}

Infertility is a complex disease and is defined as a couple's inability to have children with unprotected regular intercourse

Correspondence to: Professor Xinyi Xia, Department of Reproduction and Genetics, Institute of Laboratory Medicine, Jinling Hospital, Nanjing University School of Medicine, 305 East Zhongshan Road, Nanjing, Jiangsu 210002, P.R. China

E-mail: xiaxynju@163.com

*Contributed equally

Key words: antioxidant genes, polymorphisms, gene-gene interaction, oxidative stress, male infertility after one year; $\sim 15 \%$ of couples suffer from infertility worldwide, amounting to 48.5 million couples (1). Several causes contribute to infertility, and male infertility is responsible for $50 \%$ of the total number of cases (2). Despite there being several causes of male infertility discussed by previous studies $(3,4)$, the cause of $50 \%$ of cases of abnormal spermatogenesis are still unknown. Previous studies have shown that 30-80\% of male infertility cases are caused by the damaging effects of oxidative stress $(5,6)$.

In vitro experiments have shown that normal quantities of reactive oxygen species (ROS) are sufficient to induce mutations, capacitation and ultimately fertilization (6-10). Oxidative stress occurs when there is an imbalance between the production of ROS and the natural antioxidant defense mechanisms (11-13). Several studies have also shown that the levels of ROS in infertile sperm samples are significantly higher compared with the levels from fertile samples (14). The damaging effects of oxidative stress cause DNA damage and lead to the loss of sperm integrity and function (Fig. 1) (11). If balance between ROS production and antioxidant mechanisms is disturbed, the emergent oxidative stress results in DNA damage and affects spermatogenesis (15).

Generally, the antioxidant defense system is able to effectively neutralize ROS in the body $(16,17)$. This precise system is regulated by several enzymes, such as glutathione $S$-transferase (GST), paraoxonase, superoxide dismutase (SOD) and NAD $(\mathrm{P}) \mathrm{H}$ dehydrogenase quinone $(12,18)$. When the genes encoding these enzymes are mutated or abnormally expressed, the antioxidant system may be disrupted, resulting in damage to sperm DNA. Therefore, polymorphisms in these antioxidant genes and their gene-gene interactions have been studied to investigate a possible association with an increased risk of male infertility (19-23). However, there may be several antioxidant genes or polymorphisms that may be potential risk factors for male infertility that have not been discovered. To fill this gap, the association between seven potential functional polymorphisms in five enzyme genes (NQO1 rs1800566, SOD2 rs4880, GSTM3 rs1571858, rs3814309, rs7483, GSTM5 rs11807 and GSTP1 rs1695) in the oxidative stress pathway and the risk of male infertility in 248 cases and 310 controls were assessed. 
GSTM3 is a member of the GST family and is located on chromosome 1p13. Polimanti et al (24) showed that GST SNPs may be associated with complex diseases including male infertility and embryotoxicity. Reactive oxygen metabolites can damage the DNA of sperm, and GSTM3 may function to protect sperm through its molecular mechanism of inactivating cytotoxic substances. Thus, three SNPs of GSTM3 (rs1571858, rs3814309, rs7483) were selected and tested for any association with male infertility.

NAD(P)H: quinone oxidoreductase1 (NQO1) serves an important role in protecting against oxidative stress by functioning as a cytoplasmic2-electron reductase $(25,26)$. Several studies have reported that polymorphisms in $N Q O 1$ are associated with male infertility $(12,22,27,28)$; thus one polymorphism of $\mathrm{NQO1}$ was chosen and investigated for association with male infertility.

Ji et al (22) found a significant association between SOD2 rs4880 and male infertility. Furthermore, Yan et al (23) also found that the SOD2 rs4880 variant genotype was associated with a low level of SOD activity.

\section{Materials and methods}

Subjects and population. The present study was performed in accordance with the Declaration of Helsinki (29), and was approved by the Ethics Committee of Jinling Hospital (Nanjing, China). Written informed consent was obtained from all participants. Samples were collected from a total of 636 patients of Han-Chinese ethnicity that had been diagnosed with unexplained male factor infertility by the Laboratory Medicine, Jinling Hospital, Nanjing University School of Medicine, between April 2013 and July 2015. At least two semen analyses were performed for all patients, and those that were found to have genetic factors (chromosomal anomalies), AZF microdeletions of the $\mathrm{Y}$ chromosome, clinical factors (varicocele, cryptorchidism or orchitis) or infections were excluded from the present study. In the final analysis, 248 men with idiopathic infertility were included, including 146 men with azoospermia or severe oligozoospermia $\left(0<\right.$ sperm concentration $<5 \times 10^{6} / \mathrm{ml}$; mean age, $28.5 \pm 4.3$ years; range, $19-39$ years) and 102 men with oligozoospermia (sperm concentration: $5-15 \times 10^{6} / \mathrm{ml}$; mean age, 28.8 \pm 4.9 years; range, $19-38$ years).

A total of 310 fertile men (mean age, $28.3 \pm 4.3$ years; range, 19-40 years) were enrolled in the control group. These men had at least 1 child as reported by direct survey and lacked any history of requiring assisted reproduction technology. All of the controls were selected from the same hospital. The semen analysis for sperm concentration, motility and morphology was performed according to the World Health Organization criteria (2010) (30).

SNP selection. SNP selection as performed through extensive mining of the International HapMap Project and dbSNP. HapMap is a catalog of common genetic polymorphisms in the human genome, which describes the forms of these mutations, their location in the DNA, and their distribution within the same population and between different populations (ncbi. nlm.nih.gov/variation/tools/1000genomes). dbSNP is world's largest database for nucleotide variations, which also contains human single nucleotide variations, microsatellites, and

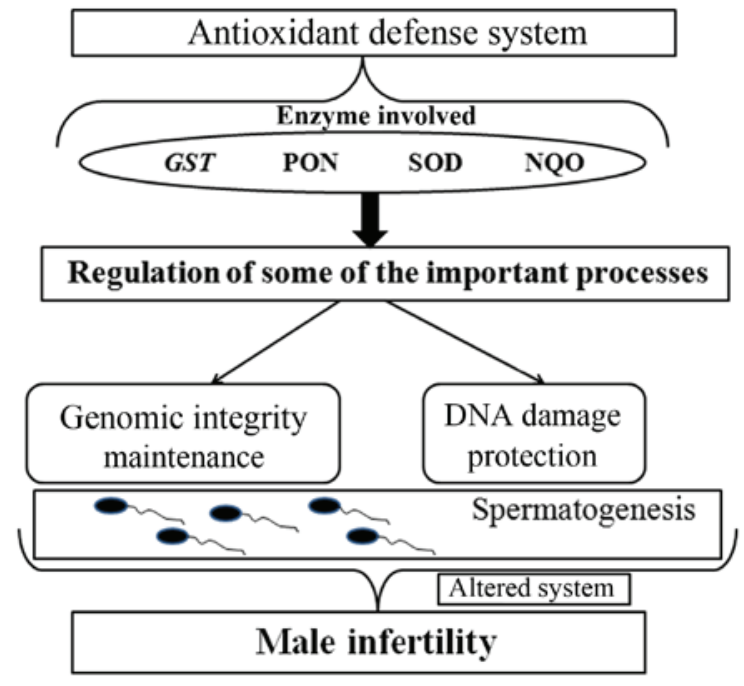

Figure 1. The antioxidant defense system and its impact on male infertility The regulatory processes involved in alterations of the antioxidant defense system results in abnormalities in spermatogenesis and may lead to infertility. GST, glutathione $S$-transferase; $P O N$, paraoxonase; $S O D$, superoxide dismutase; $N Q O, \mathrm{NAD}(\mathrm{P}) \mathrm{H}$ dehydrogenase quinone.

small-scale insertions and deletions along with the original publications (ncbi.nlm.nih.gov/SNP/). A total of 7 potential functional polymorphisms were identified in the genes of five enzyme involved in oxidative stress response.

Genomic DNA extraction and genotyping. Genomic DNA was extracted from the leukocytes in venous blood from each patient and controls using a blood DNA extraction kit (Tiangen Biotech Co., Ltd.). The DNA was purified using a Genomic DNA Purification kit (DU530UV/VIS spectrophotometer; Beckman Coulter, Inc.). Genotyping was performed using the Mass ARRAY platform. Briefly, SNPs were detected using a Sequenom Mass ARRAY RS1000 according to the manufacturer's protocol. The multiplexed SNP Mass EXTENDED assay was designed by Sequenom Mass ARRAY Assay Design software version 3.0 (Sequenom). Data management and analysis were performed using a Sequenom Mass ARRAY Analyzer 4.0 system (Sequenom).

Statistical analysis. The expected frequencies of genotypes in the control group were tested for Hardy-Weinberg equilibrium (HWE) using the exact test. The allele and genotype frequencies of the controls and patients were directly calculated by counting. To examine the associations between genetic polymorphisms and male infertility, the odds ratio (OR) and $95 \%$ confidence intervals (CI) were calculated using a logistic regression model with SPSS version 11.0 (SPSS, Inc.). Two-tailed $\mathrm{P}<0.05$ was considered to indicate a statistically significant difference. Haplotype analysis was performed using SHEsis (analysis.bio-X.cn/myAnalysis.php).

\section{Results}

Clinical characteristics of the study population. The present case-controlled study contained 248 infertility patients and 310 fertile controls. The position and minor allele frequency of the seven functional SNPs found in the Chinese population in 
Table I. Primary information on the seven assessed SNPs in antioxidant genes.

\begin{tabular}{llc}
\hline Gene: SNP & Location or amino acid change & MAF in Chinese population $^{\mathrm{a}}$ \\
\hline NQO1: rs1800566 C-T & nsSNP/P187S & 0.5 \\
SOD2: rs4880 T-C & nsSNP/V16A & 0.117 \\
GSTM3: rs1571858 A-G & Intronic & 0.252 \\
GSTM3: rs3814309 C-T & 3'-UTR & 0.243 \\
GSTM3: rs7483 A-G & nsSNP/V224I & 0.243 \\
GSTM5 rs11807 A-G & 3'-UTR & 0.146 \\
GSTP1: rs1695 A-G & nsSNP/I105V & 0.185
\end{tabular}

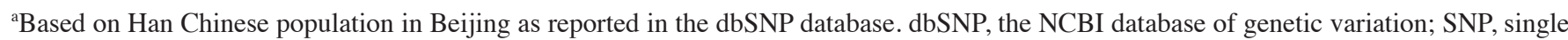
nucleotide polymorphism; MAF, minimum allele frequency; nsSNP, nonsynonymous SNP; UTR, untranslated region.

Table II. Distribution of the control and case groups by genotype.

\begin{tabular}{|c|c|c|c|c|c|}
\hline Genotype & All cases & Azoospermia & Oligozoospermia & Control & $\mathrm{P}_{\text {HWE }}$ \\
\hline rs4880 & & & & & 0.086 \\
\hline TT:TC:CC & $188: 55: 05$ & $112: 32: 02$ & $76: 23: 03$ & 240:69:1 & \\
\hline MAF & $13 \%$ & $22 \%$ & $25 \%$ & $11.50 \%$ & \\
\hline rs 1571858 & & & & & 0.576 \\
\hline AA:AG:GG & $139: 92: 17$ & $81: 53: 12$ & $58: 39: 05$ & 174:114:22 & \\
\hline MAF & $26 \%$ & $26 \%$ & $24 \%$ & $26 \%$ & \\
\hline rs3814309 & & & & & 0.733 \\
\hline CC:CT:TT & $139: 92: 17$ & $81: 53: 12$ & $58: 39: 05$ & 180:111:19 & \\
\hline MAF & $26 \%$ & $26 \%$ & $24 \%$ & $24 \%$ & \\
\hline rs 7483 & & & & & 0.798 \\
\hline AA:AG:GG & $142: 89: 17$ & $82: 52: 12$ & $60: 37: 05$ & $82: 110: 18$ & \\
\hline MAF & $25 \%$ & $26 \%$ & $23 \%$ & $24 \%$ & \\
\hline rs11807 & & & & & 0.642 \\
\hline AA:AG:GG & $176: 66: 6$ & 103:41:02 & $73: 25: 04$ & 0.97302083 & \\
\hline MAF & $16 \%$ & $15 \%$ & $16 \%$ & $16 \%$ & \\
\hline rs1695 & & & & & 0.729 \\
\hline AA:AG:GG & $147: 95: 6$ & $85: 58: 03$ & $62: 37: 03$ & 197:99:14 & \\
\hline MAF & $22 \%$ & $22 \%$ & $21 \%$ & $21 \%$ & \\
\hline rs1800566 & & & & & 0.43 \\
\hline TT:CT:CC & $54: 124: 70$ & $33: 76: 37$ & $33: 48: 21$ & $84: 148: 78$ & \\
\hline MAF & $53 \%$ & $37 \%$ & $44 \%$ & $49 \%$ & \\
\hline
\end{tabular}

MAF: minor allele frequency; P-value for Hardy-Weinberg Equilibrium. Ratios; genotypes of the control and case groups: (azoospermia or severe oligozoospermia and oligozoospermia): MAF.

the HapMap database are presented in Table I. The genotypes of seven polymorphisms in antioxidant genes were determined in the control group and case groups (azoospermia or severe oligozoospermia and oligozoospermia; Table II). The genotype frequencies of all investigated polymorphisms were found to be in HWE in all of the control groups.

Association between polymorphisms in oxidative stress genes and risk of male infertility. The associations of polymorphisms in oxidative stress genes and risk of male infertility are shown in Table III. There were no significant associations found between the seven polymorphisms and risk of male infertility using a logistic regression model. Haplotype analysis for the three GSTM3 SNPs was performed; there were no significant associations between these three SNPs and male infertility. It was hypothesized that gene-gene interactions may contribute to male infertility. To test this hypothesis, statistical analysis of gene-gene interactions between NQO1, SOD2, GSTM3, GSTM5 and GSTP1 was performed. There was a decreased risk in male infertility with a gene-gene interaction between 
Table III. Association between SNPs in antioxidant genes and risk of male infertility in subjects.

\begin{tabular}{|c|c|c|c|c|c|c|c|c|c|}
\hline \multirow[b]{2}{*}{ SNP } & \multicolumn{3}{|c|}{ All subjects, $n=558$} & \multicolumn{3}{|c|}{ Azoospermia, $n=456$} & \multicolumn{3}{|c|}{ Oligozoospermia, $n=412$} \\
\hline & $\mathrm{n}$ & P-value & OR $(95 \% \mathrm{CI})$ & $\mathrm{n}$ & P-value & OR $(95 \% \mathrm{CI})$ & $\mathrm{n}$ & P-value & OR $(95 \% \mathrm{CI})$ \\
\hline \multicolumn{10}{|c|}{ GSTP1 rs1695 } \\
\hline AA & 344 & 0.161 & Ref & 282 & 0.155 & Ref & 259 & 0.611 & Ref \\
\hline $\mathrm{AG}$ & 194 & 0.163 & $1.29(0.92-1.83)$ & 157 & 0.146 & $1.36(0.90-2.05)$ & 136 & 0.477 & $1.19(0.74-1.91)$ \\
\hline GG & 20 & 0.267 & $0.57(0.22-1.53)$ & 17 & 0.281 & $0.50(0.14-1.77)$ & 17 & 0.556 & $0.68(0.19-2.45)$ \\
\hline AG/GG & 214 & 0.302 & $1.20(0.85-1.69)$ & 174 & 0.275 & $1.25(0.84-1.87)$ & 153 & 0.616 & $1.13(0.71-1.78)$ \\
\hline \multicolumn{10}{|c|}{ GSTM3 rs1571858 } \\
\hline AA & 313 & 0.992 & Ref & 255 & 0.914 & Ref & 232 & 0.738 & Ref \\
\hline $\mathrm{AG}$ & 206 & 0.955 & $1.01(0.71-1.44)$ & 167 & 0.995 & $0.99(0.66-1.52)$ & 153 & 0.914 & $1.03(0.64-1.64)$ \\
\hline GG & 39 & 0.923 & $0.97(0.49-1.89)$ & 34 & 0.679 & $1.17(0.55-2.48)$ & 27 & 0.46 & $0.68(0.24-1.88)$ \\
\hline AG/GG & 245 & 0.985 & $1.00(0.72-1.41)$ & 201 & 0.896 & $1.03(0.69-1.53)$ & 180 & 0.897 & $0.97(0.62-1.53)$ \\
\hline \multicolumn{10}{|c|}{ SOD2 rs4880 } \\
\hline $\mathrm{TT}$ & 428 & 0.242 & Ref & 352 & 0.495 & Ref & 316 & 0.153 & Ref \\
\hline $\mathrm{TC}$ & 124 & 0.932 & $1.02(0.68-1.52)$ & 101 & 0.98 & $0.99(0.62-1.60)$ & 92 & 0.743 & $0.93(0.60-1.43)$ \\
\hline $\mathrm{CC}$ & 6 & 0.92 & $6.84(0.74-55.10)$ & 3 & 0.237 & $4.29(0.39-47.76)$ & 4 & 0.147 & $5.37(0.55-52.11)$ \\
\hline $\mathrm{TC} / \mathrm{CC}$ & 130 & 0.654 & $1.09(0.74-1.62)$ & 104 & 0.867 & $1.04(0.65-1.66)$ & 96 & 0.952 & $0.99(0.65-1.51)$ \\
\hline \multicolumn{10}{|c|}{ GSTM5 rs 11807} \\
\hline AA & 396 & 0.936 & Ref & 323 & 0.587 & Ref & 293 & 0.447 & Ref \\
\hline $\mathrm{AG}$ & 147 & 0.925 & $1.02(0.70-1.49)$ & 122 & 0.73 & $1.08(0.70-1.68)$ & 106 & 0.211 & $0.76(0.50-1.17)$ \\
\hline GG & 15 & 0.734 & $0.83(0.29-2.39)$ & 11 & 0.346 & $0.48(0.10-2.24)$ & 13 & 0.75 & $0.84(0.28-2.50)$ \\
\hline $\mathrm{AG} / \mathrm{GG}$ & 162 & 1 & $1.00(0.69-1.45)$ & 133 & 0.927 & $1.02(0.66-1.57)$ & 119 & 0.208 & $0.77(0.51-1.16)$ \\
\hline \multicolumn{10}{|c|}{ GSTM3 rs7483 } \\
\hline AA & 324 & 0.862 & Ref & 264 & 0.612 & Ref & 242 & 0.618 & Ref \\
\hline $\mathrm{AG}$ & 199 & 0.841 & $1.04(0.73-1.48)$ & 162 & 0.823 & $1.05(0.69-1.60)$ & 147 & 0.991 & $0.99(0.68-1.47)$ \\
\hline GG & 35 & 0.592 & $1.21(0.60-2.43)$ & 30 & 0.322 & $1.48(0.68-3.21)$ & 23 & 0.339 & $1.40(0.70-2.80)$ \\
\hline AG/GG & 234 & 0.73 & $1.06(0.76-1.49)$ & 192 & 0.608 & $1.11(0.75-1.65)$ & 170 & 0.758 & $1.06(0.74-1.52)$ \\
\hline \multicolumn{10}{|c|}{ GSTM3 rs3814309 } \\
\hline $\mathrm{CC}$ & 319 & 0.872 & Ref & 261 & 0.685 & Ref & 238 & 0.7 & Ref \\
\hline $\mathrm{CT}$ & 203 & 0.695 & $1.07(0.75-1.53)$ & 164 & 0.782 & $1.06(0.70-1.61)$ & 150 & 0.984 & $1.00(0.68-1.48)$ \\
\hline $\mathrm{TT}$ & 36 & 0.676 & $1.16(0.58-2.31)$ & 31 & 0.387 & $1.40(0.65-3.03)$ & 24 & 0.407 & $1.34(0.67-2.66)$ \\
\hline $\mathrm{CT} / \mathrm{TT}$ & 239 & 0.633 & $1.09(0.78-1.52)$ & 195 & 0.603 & $1.11(0.75-1.65)$ & 174 & 0.768 & $1.06(0.74-1.52)$ \\
\hline \multicolumn{10}{|c|}{ NQO1 rs1800566 } \\
\hline $\mathrm{CC}$ & 148 & 0.332 & Ref & 115 & 0.561 & Ref & 111 & 0.699 & Ref \\
\hline $\mathrm{CT}$ & 272 & 0.737 & $0.93(0.63-1.40)$ & 224 & 0.746 & $1.08(0.67-1.74)$ & 196 & 0.293 & $0.94(0.62-1.45)$ \\
\hline TT & 138 & 0.164 & $0.72(0.45-1.15)$ & 117 & 0.51 & $0.83(0.47-1.45)$ & 105 & 0.413 & $0.88(0.49-1.34)$ \\
\hline $\mathrm{CT} / \mathrm{TT}$ & 410 & 0.415 & $0.86(0.59-1.25)$ & 341 & 0.967 & $0.99(0.63-1.58)$ & 301 & 0.598 & $0.90(0.60-1.34)$ \\
\hline
\end{tabular}

OR, odds ratio; CI, confidence interval; Ref, reference genotype.

GSTM3 rs3814309 and NQO1 rs1800566 (CC x CT/TT; $\mathrm{OR}=0.55,95 \% \mathrm{CI}=0.34-0.92 ; \mathrm{P}=0.022$; Table IV). There was also a significant association between gene-gene interactions of GSTM3 rs1571858 and NQO1 rs1800566 and azoospermia (AG/GG x CC; $\mathrm{OR}=0.42,95 \% \mathrm{CI}=0.18-0.97 ; \mathrm{P}=0.043)$.

\section{Discussion}

Male infertility is a complex disease that is caused by several factors (31-34). Sperm DNA integrity and expression is regulated by a precise system in body, and any damage to sperm DNA may result in spermatogenesis failure and thus male infertility. Previously, several studies have shown that oxidative stress is associated with male infertility through damaging sperm DNA $(7,35)$. SNPs have been shown to be associated with the activities of antioxidant defense system enzymes. In the present study, a case-controlled study to investigate the association of seven SNPs (NQO1 rs1800566, SOD2 rs4880, GSTM3 rs1571858, rs3814309, rs7483, GSTM5 rs11807 and GSTP1 rs1695) in antioxidant genes with male 
Table IV. Association between SNPs in antioxidant gene-gene interactions and male infertility risk.

\begin{tabular}{|c|c|c|c|c|c|c|}
\hline \multirow{2}{*}{$\begin{array}{l}\text { Groups } \\
\text { All subjects, } n=558\end{array}$} & \multirow{2}{*}{$\frac{\text { Gene-Gene }}{\text { rs3814309/rs } 1800566}$} & \multicolumn{2}{|c|}{ Genotypes } & \multirow{2}{*}{$\begin{array}{c}\mathrm{n} \\
87\end{array}$} & \multirow{2}{*}{$\frac{\text { OR }(95 \% \mathrm{CI})}{\text { Ref }}$} & \multirow{2}{*}{$\begin{array}{c}\text { P-value } \\
0.058\end{array}$} \\
\hline & & $\mathrm{CC}$ & $\mathrm{CC}$ & & & \\
\hline & & $\mathrm{CC}$ & $\mathrm{CT} / \mathrm{TT}$ & 232 & $0.55(0.34-0.92)$ & 0.022 \\
\hline & & $\mathrm{CT} / \mathrm{TT}$ & $\mathrm{CT} / \mathrm{TT}$ & 178 & $0.80(0.48-1.33)$ & 0.085 \\
\hline & & $\mathrm{CT} / \mathrm{TT}$ & $\mathrm{CC}$ & 61 & $0.52(0.26-1.00)$ & 0.052 \\
\hline \multirow[t]{4}{*}{ Azoospermia, $n=456$} & rs $1571858 /$ rs 1800566 & $\mathrm{AA}$ & $\mathrm{CC}$ & 65 & Ref & 0.116 \\
\hline & & AA & $\mathrm{CT} / \mathrm{TT}$ & 190 & $0.61(0.34-1.01)$ & 0.1 \\
\hline & & AG/GG & $\mathrm{CT} / \mathrm{TT}$ & 161 & $0.84(0.46-1.52)$ & 0.554 \\
\hline & & $\mathrm{AG} / \mathrm{GG}$ & $\mathrm{CC}$ & 50 & $0.42(0.18-0.97)$ & 0.043 \\
\hline
\end{tabular}

OR, odds ratio; CI, confidence interval; Ref, reference genotype.

infertility in Chinese individuals was performed. The results showed there were no associations between the seven SNPs and male infertility. Haplotype analysis of three GSTM3 SNPs and gene-gene interaction analysis of the seven SNPs was also performed. There were no significant associations identified using haplotype analysis; however there was a decreased risk for male infertility with a gene-gene interaction between GSTM3 rs3814309 and NQO1 rs1800566 (CC x CT/TT) in all subjects and a significant interaction between GSTM3 rs1571858 and NQO1 rs1800566 (AG/GG x CC) in azoospermia.

GSTM3 rs1571858 is located in the intronic region, and rs3814309 is located on the 3'-untranslated region of GSTM3. However, no previous studies have investigated the effect of rs3814309 and rs1571858 on GSTM3 function, to the best of our knowledge. rs7483 is a missense mutation, which results in a substitution of the expected Val amino acid with Ile. There was no association between the three SNPs (rs1571858, rs3814309 and rs7483) of GSTM3 and a risk of male infertility. However, a gene-gene interaction between GSTM3 rs3814309 and NQO1 rs1800566 (CC x CT/TT) was associated with a decreased risk for male infertility in all subjects. In the present study, the genotype RR (homozygous mutation) was of low abundance in the case group, which may have affected the true result. Therefore, a larger sample size of patients with the three SNPs (rs3814309, rs1800566 and rs7483) who suffer from male infertility is required to confirm these results.

NQO1 rs1800566 is a missense mutation (P187S), which is hypothesized to influence the enzymatic activity and concentration of NQO1. However, there was no association between NQO1 rs1800566 and risk of male infertility. This result is consistent with a previous report by Ji et al (22), who also found no significant association between this polymorphism and male infertility, suggesting that NQO1 rs1800566 is not a risk factor for male infertility. A gene-gene interaction between GSTM3 rs1571858 and NQO1 rs1800566 (AG/GG x CC) was a significant factor for male infertility in patients with azoospermia. Although the rs1571858 SNP is located in the intronic region of GSTM3, it was hypothesized that rs1571858 may affect GSTM3 function via an unknown mechanism.

The present study has some limitations. Gene-environment interaction analysis for male infertility was not performed. The lifestyles of patients including smoking, drinking or other potentially detrimental habits contribute to male infertility.
Additionally, the demographic characteristics of enrolled participants, including clinical data, profession, health status index are not presented. As in all case-controlled studies, a selection bias may exist, which may influence the discovery of real associations. Finally, the association of polymorphisms in antioxidant genes and male infertility in other ethnicities were not assessed. Therefore, any associations, or lack thereof, demonstrated in the present study, should be confirm with a larger more diverse cohort.

\section{Acknowledgements}

We would like to thank Dr Bangshun He, Nanjing First Hospital for their expert advice on data analysis.

\section{Funding}

This work was supported by Nanjing Science and Technology Planning Project (grant no. 201605004), Jangsu Science and Technology Planning Project (grant no. BE2018713), The Central Military Commission Logistics Support Department, The Military Family Planning Special Research Task Plan Key Projects (grant no. 18JS005), and The Subject Of Nanjing General Hospital, IHH new pathogenic locus identification and pathogenesis research (grant no. 2017046).

\section{Availability of data and materials}

The datasets used and/or analyzed during the present study are available from the corresponding author on reasonable request.

\section{Authors' contributions}

XX conceived and designed the present study. YY and PZ analyzed the data and wrote the manuscript. TL collected the samples. All authors read and approved the final manuscript.

\section{Ethics approval and consent to participate}

The present study was approved by the Ethics Committee of Jinling Hospital (Nanjing, China). All patients provided written informed consent. 


\section{Patient consent for publication}

Not applicable.

\section{Competing interests}

The authors declare that they have no competing interests.

\section{References}

1. Agarwal A, Mulgund A, Hamada A and Chyatte MR: A unique view on male infertility around the globe. Reprod Biol Endocrinol 13: 37, 2015.

2. Sato Y, Tajima A, Tsunematsu K, Nozawa S, Yoshiike M, Koh E, Kanaya J, Namiki M, Matsumiya K, Tsujimura A, et al: An association study of four candidate loci for human male fertility traits with male infertility. Hum Reprod 30: 1510-1514, 2015.

3. Esteves SC, Miyaoka R and Agarwal A: An update on the clinical assessment of the infertile male [corrected]. Clinics (Sao Paulo) 66: 691-700, 2011.

4. Hamada AJ, Esteves SC and Agarwal A: A comprehensive review of genetics and genetic testing in azoospermia. Clinics (Sao Paulo) 68(Suppl 1): 39-60, 2013.

5. Tremellen K: Oxidative stress and male infertility-a clinical perspective. Hum Reprod update 14: 243-258, 2008.

6. Ko EY, Sabanegh ES Jr and Agarwal A: Male infertility testing: Reactive oxygen species and antioxidant capacity. Fertil Steril 102: 1518-1527, 2014.

7. Wright C, Milne S and Leeson H: Sperm DNA damage caused by oxidative stress: Modifiable clinical, lifestyle and nutritional factors in male infertility. Reprod Biomed Online 28: 684-703, 2014.

8. Agarwal A and Allamaneni SS: Role of free radicals in female reproductive diseases and assisted reproduction. Reprod Biomed Online 9: 338-347, 2004.

9. De Lamirande E and Cagnon C: Human sperm hyperactivation and capacitation as parts of an oxidative process. Free Radic Biol Med 14: 157-166, 1993.

10. Tremellen K, Miari G, Froiland D and Thompson J: A randomised control trial examining the effect of an antioxidant (Menevit) on pregnancy outcome during IVF-ICSI treatment. Aust N Z J Obstet Gynaecol 47: 216-221, 2007.

11. Said TM, Agarwal A, Sharma RK, Thomas AJ Jr and Sikka SC: Impact of sperm morphology on DNA damage caused by oxidative stress induced by beta-nicotinamide adenine dinucleotide phosphate. Fertil Steril 83: 95-103, 2005.

12. Ichioka K, Nagahama K, Okubo K, Soda T, Ogawa O and Nishiyama $\mathrm{H}$ : Genetic polymorphisms in glutathione $\mathrm{S}$-transferase T1 affect the surgical outcome of varicocelectomies in infertile patients. Asian J Androl 11: 333-341, 2009.

13. Pisoschi AM and Pop A: The role of antioxidants in the chemistry of oxidative stress: A review. Eur J Med Chem 97: 55-74, 2015.

14. Guz J, Gackowski D, Foksinski M, Rozalski R, Zarakowska E, Siomek A, Szpila A, Kotzbach M, Kotzbach R and Olinski R: Comparison of oxidative stress/DNA damage in semen and blood of fertile and infertile men. PLoS One 8: e68490, 2013.

15. Shen $\mathrm{H}$ and Ong C: Detection of oxidative DNA damage in human sperm and its association with sperm function and male infertility. Free Radic Biol Med 28: 529-536, 2000.

16. Amir Aslani B and Ghobadi S: Studies on oxidants and antioxidants with a brief glance at their relevance to the immune system. Life Sci 146: 163-173, 2016.

17. Reszka E and Wasowicz W: Significance of genetic polymorphisms in glutathione S-transferase multigene family and lung cancer risk. Int J Occup Med Environ Health 14: 99-113, 2001.
18. Forsberg L, de Faire U and Morgenstern R: Oxidative stress, human genetic variation, and disease. Arch Biochem Biophys 389: 84-93, 2001.

19. Jun S, Pierce A and Dory L: Extracellular superoxide dismutase polymorphism in mice: Allele-specific effects on phenotype. Free Radic Biol Med 48: 590-596, 2010.

20. Gallagher CJ, Ahn K, Knipe AL, Dyer AM, Richie JP Jr, Lazarus P and Muscat JE: Association between haplotypes of manganese superoxide dismutase (SOD2), smoking, and lung cancer risk. Free Radic Biol Med 46: 20-24, 2009.

21. Nejatizadeh A, Kumar R, Stobdan T, Goyal AK, Sikdar S, Gupta M, Javed S and Pasha MA: Endothelial nitric oxide synthase gene haplotypes and circulating nitric oxide levels significantly associate with risk of essential hypertension. Free Radic Biol Med 44: 1912-1918, 2008.

22. Ji G, Gu A, Wang Y, Huang C, Hu F, Zhou Y, Song L and Wang X: Genetic variants in antioxidant genes are associated with sperm DNA damage and risk of male infertility in a Chinese population. Free Radic Biol Med 52: 775-780, 2012.

23. Yan L, Liu J, Wu S, Zhang S, Ji G and Gu A: Seminal superoxide dismutase activity and its relationship with semen quality and SOD gene polymorphism. J Assist Reprod Genet 31: 549-554, 2014.

24. Polimanti R, Piacentini S and Fuciarelli M: HapMap-based study of human soluble glutathione $S$-transferase enzymes: The role of natural selection in shaping the single nucleotide polymorphism diversity of xenobiotic-metabolizing genes. Pharmacogenet Genomics 21: 665-672, 2011.

25. Yang FY, Guan QK, Cui YH, Zhao ZQ, Rao W and Xi Z: NAD(P)H quinone oxidoreductase 1 (NQO1) genetic C609T polymorphism is associated with the risk of digestive tract cancer: A meta-analysis based on 21 case-control studies. Eur J Cancer Prev 21: 432-441, 2012.

26. Han FF, Guo CL, Gong LL, Jin Z and Liu LH: Effects of the NQO1 609C $>$ T polymorphism on leukemia susceptibility: Evidence from a meta-analysis. Asian Pac J Cancer Prev 14: 5311-5316, 2013.

27. Corrales J, Fang X, Thornton C, Mei W, Barbazuk WB, Duke M, Scheffler BE and Willett KL: Effects on specific promoter DNA methylation in zebrafish embryos and larvae following benzo[a]pyrene exposure. Comp Biochem Physiol C Toxicol Pharmacol 163: 37-46, 2014.

28. Zappa F, Ward T, Butler J, Pedrinis E and Mcgown A: Overexpression of NAD(P)H:quinone oxidoreductase 1 in human reproductive system. J Histochem Cytochem 49: 1187-1188, 2001.

29. Goodyear MD, Krleza-Jeric K and Lemmens T: The declaration of Helsinki. BMJ 335: 624-625, 2007.

30. World Health Organization (WHO): World Health Statistics 2010. Fact sheet $\mathrm{N}^{\circ} 290$. WHO, Geneva, 2010.

31. Miyamoto T, Minase G, Okabe K, Ueda H and Sengoku K: Male infertility and its genetic causes. J Obstet Gynaecol Res 41: 1501-1505, 2015.

32. Oliva A, Spira A and Multigner L: Contribution of environmental factors to the risk of male infertility. Hum Reprod 16: 1768-1776, 2001.

33. Zhang S, Tang Q, Wu W, Yuan B, Lu C, Xia Y, Ding H, Hu L, Chen D, Sha J and Wang X: Association between DAZL polymorphisms and susceptibility to male infertility: Systematic review with meta-analysis and trial sequential analysis. Sci Rep 4: 4642, 2014.

34. Jiang W, Sun H, Zhang J, Zhou Q, Wu Q, Li T, Zhang C, Li W, Zhang $\mathrm{M}$ and Xia X: Polymorphisms in Protamine 1 and Protamine 2 predict the risk of male infertility: A meta-analysis. Sci Rep 5: 153000, 2015.

35. Hosen MB, Islam MR, Begum F, Kabir Y and Howlader MZH: Oxidative stress induced sperm DNA damage, a possible reason for male infertility. Iran J Reprod Biomed 13: 525-532, 2015. 